\title{
DEVELOPMENT AND IMPLEMENTATION OF A ADAPTIVE FUZZY CONTROL SYSTEM FOR A VTOL VEHICLE IN HOVERING MODE
}

\author{
Ehsan. Abbasi \\ Department of Mechanical Engineering, Isfahan University of Technology, Isfahan, Iran
}

\begin{abstract}
The studies in aerial vehicles modeling and control have been increased rapidly recently. This paper presents the modeling and control of a four rotor vertical take-off and landing (VTOL) vehicle. The modeling of the VTOL vehicle will be described by using Euler-Newton equations. In order to stable this vehicle and control the attitude of that, classical PID controller and a fuzzy system that adjusts the PID controller gains, have been designed. Although fuzzy control of various dynamical systems has been presented in literature, application of this technology to aerial vehicle control is quite new. This system has nonlinear characteristics where classical control methods are not adequate for stabilize that. On the other hand, fuzzy control is nonlinear and it is thus suitable for nonlinear system control. Matlab Simulink has been used to test, analyze and compare the performance of the controllers in simulations. Finally this presented controller will be implemented on a real vehicle and performance of that will be showed. This study showed that although, both of the classical PID and the fuzzy self-tuning PID controllers, can control the system properly, the second controller performed better than the classical PID controller.
\end{abstract}

\section{KEYWORDS}

VTOL, Fuzzy control, Modeling, Attitude control, PID controller.

\section{INTRODUCTION}

unmanned aerial vehicles has attracted a great amount of attention among scientists over the last decades, due to, the widespread area of applications, e.g. near-area surveillance, crop dusting firefighting, exploration both in military and commercial in- and outdoor applications, and so on. Helicopter design has been the center of attention since the beginning of the 20th century. First full-scale four rotor helicopter (quadrotor) was built by Debothezat in 1921 [1]. Other examples are Breguet Richet helicopter, Oemnichen helicopter, Convertawings Model A and Curtis Wright VZ-7 [2,3]. At those early times due to the lacking control and sensing technologies, it was not possible to build an unmanned vehicle. Advances in sensors, control technology and electronics enable the possibility of that. Currently, there are various commercial and experimental autonomous unmanned VTOL vehicles are being developed at universities, research centers, and by hobbists [4-7].

The studies in quadrotor modeling and control have been increased rapidly recently. A number of examples of these studies can be summarized as following; Altuğ et al. modeled a quadrotor using Euler-Newton method and worked on vision based stabilization and output tracking control [8]. Suter et al. also studied on image based visual servo control for quadrotors [9]. Dunfied et al. created a neural networks controller for a quadrotor [10]. Earl et al. used a Kalman filter to estimate the attitude of a quadrotor [11]. Lee et al. presented feedback linearization vs. adaptive sliding mode control for a quadrotor helicopter [12]. Bora et al. used a hybrid fuzzy logic 
controller for a quadrotor [13]. Astha et al. presented a fuzzy logic controller for a quadrotor and compared it to conventional PID controller [14].

The fuzzy logic control is an active field in last couple of decades, and it has been implemented on various dynamical systems [15-20]. The fuzzy logic has also been implemented in helicopter control [21-23]. In more recent papers [24,25], the researcher used a fuzzy controller for the altitude and hovering control of an unmanned helicopter. Although fuzzy control of dynamical systems has seen presented extensively in literature, application of this to quadrotor helicopter control is quite new.

Although the quadrotor has the advantages in easy mechanical construction against the traditional helicopter, but there are still issues that prevent it from being widely used in many of the suggested fields and applications. For example, the stabilizing control and guidance of the quadrotor is a difficult task because of the nonlinear dynamic behavior. Conventional control methods use linear theory that is suitable for linear systems only. Fuzzy control is nonlinear and it is thus suitable for nonlinear system control.

This paper will be presented a fuzzy system for control the quadrotor. Additionally, detailed model of the quadrotor is given. The performances of the fuzzy controllers are compared to classical PID controllers using Matlab simulations. And in real world this controller will be tested on the real quadrotor and performance of that will be showed.

The paper is structured as followings: In section 2 the mathematical model of the quadrotor is described. The controllers are presented in section 3. The simulations supporting the objectives of the paper are presented in section 4. Results of the implementation of the presented controller will be showed in section 5. And finally concluding remarks are presented in section 6 .

\section{Mathematical Model OF The Quadrotor}

Generally, the quadrotor can be modeled with a four rotors in cross configuration. The throttle movement is provided by increasing (or decreasing) all the rotor speeds by the same amount. It leads a vertical force $\mathrm{u} 1(\mathrm{~N})$ with respect to body-fixed frame which raises or lowers the quadrotor.

The roll movement is provided by increasing (or decreasing) the left rotor's speed and at the same time decreasing (or increasing) the right rotor's speed. The pitch movement is provided by a same way but with other two motors. The front and rear motors rotate counter-clockwise while other two motors rotate clockwise, so yaw command is derived by increasing (or decreasing) counterclockwise motors speed and at the same time decreasing (or increasing) clockwise motor speeds.

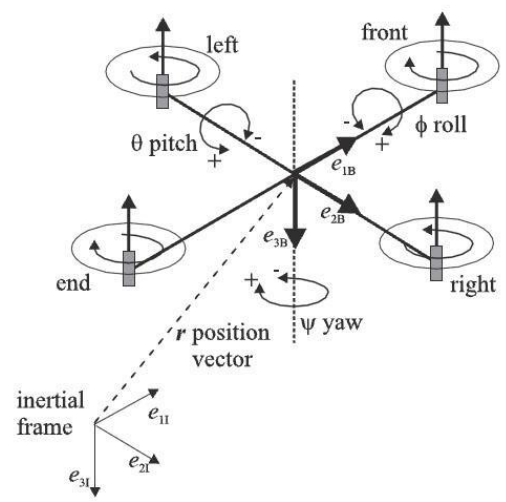

Figure 1. Configuration, inertial and body fixed frame [26]. 
In order to model the quadrotor dynamics, two frames have to be defined as showed in figure. 1. The orientation of the quadrotor is given by the three Euler angles, which are roll angle $\varphi$, pitch angle ${ }^{\theta}$ and yaw angle $\psi$.

These three Euler angles form the vector $\Omega^{T}=(\varphi, \theta, \psi)$. The position of the vehicle in the inertial frame is given by the vector $\mathrm{r}^{T}=(x, y, z)$. The transformation of vectors from the body fixed frame to the inertial frame is given by the rotation matrix $\mathrm{R}$ where ${ }^{c \theta}$ for example denotes $\cos \theta$ and $s \theta$ denotes $\sin \theta$.

$$
R=\left(\begin{array}{ccc}
c \psi \cdot c \theta & c \psi \cdot s \theta \cdot s \varphi-s \psi \cdot c \varphi & c \psi \cdot s \theta \cdot c \varphi+s \psi \cdot s \varphi \\
s \psi \cdot c \theta & s \psi \cdot s \theta \cdot s \varphi+c \psi \cdot c \varphi & s \psi \cdot s \theta \cdot c \varphi-c \psi \cdot s \varphi \\
-s \theta & c \theta \cdot s \varphi & c \theta \cdot c \varphi
\end{array}\right)
$$

The thrust force generated by rotor $i, i=1,2,3,4$ is $F_{i}=b \cdot \omega_{i}^{2}$, where ${ }^{b}$ is the thrust factor and $\omega_{i}(\mathrm{rad} / \mathrm{s})$ is the rotational speed of rotor ${ }^{i}$. Then the thrust force applied to the airframe from the four rotors is given by

$$
T=\sum_{i=1}^{4}\left|F_{i}\right|=b \cdot \sum_{i=1}^{4} \omega_{i}^{2}
$$

Now the first set of differential equation that describes the acceleration of the quadrotor can be written as:

$$
\left(\begin{array}{l}
\ddot{x} \\
\ddot{y} \\
\ddot{z}
\end{array}\right)=g \cdot\left(\begin{array}{l}
0 \\
0 \\
1
\end{array}\right)-R \frac{T}{m} \cdot\left(\begin{array}{l}
0 \\
0 \\
1
\end{array}\right)
$$

With inertia matrix ${ }^{I}$ (which is diagonal matrix with the inertias $I_{x,} I_{y}$ and $I_{z}$ on the main diagonal), the rotor inertia $I_{R}$, the vector ${ }^{M}$ that describes the torque applied to the vehicle's body and the vector $M_{G}$ of the gyroscopic torques we obtain a second set of differential equations:

$$
I . \tilde{\Omega}=-(\hat{\Omega} \times I . \dot{\Omega})-M_{G}+M
$$

The vector ${ }^{M}$ is defined as:

$$
M=\left(\begin{array}{c}
L b\left(\omega_{3}^{2}-\omega_{4}^{2}\right) \\
L b\left(\omega_{1}^{2}-\omega_{2}^{2}\right) \\
d\left(\omega_{1}^{2}+\omega_{2}^{2}-\omega_{3}^{2}-\omega_{4}^{2}\right)
\end{array}\right)
$$

The four rotational velocities $\omega_{i}$ of the rotors are the input variables of the real vehicle, but with regard to the obtained model a transformation of the input is suitable. Therefore, the artificial input variables can be defined as follows:

$$
\begin{aligned}
& u_{1}=b\left(\omega_{1}^{2}+\omega_{2}^{2}+\omega_{3}^{2}+\omega_{4}^{2}\right) \\
& u_{2}=b\left(\omega_{3}^{2}-\omega_{4}^{2}\right) \\
& u_{3}=b\left(\omega_{1}^{2}-\omega_{2}^{2}\right)
\end{aligned}
$$




$$
u_{4}=d\left(\omega_{1}^{2}+\omega_{2}^{2}-\omega_{3}^{2}-\omega_{4}^{2}\right)
$$

Where $u_{1}=T_{(6)}$ denotes the thrust force applied to the quadrotor airframe; $u_{2}$ denotes the force which leads to the roll torque; $u_{3}$ for the pitch torque and $u_{4}$ for the yaw torque.

However, also the gyroscopic torques depend on the rotational velocities of the rotors and hence on the vector $u^{T}=\left(u_{1}, u_{2}, u_{3}, u_{4}\right)$ of the transformed input variables. We assume that:

$$
g(u)=\omega_{1}+\omega_{2}-\omega_{3}-\omega_{4}
$$

And then evaluation of (4) and (5) yields the overall dynamic model in the following form:

$$
\begin{aligned}
& \ddot{x}=-(\cos \varphi \cdot \sin \theta \cdot \cos \psi+\sin \varphi \cdot \sin \psi) \cdot \frac{u_{1}}{m} \\
& \ddot{y}=-(\cos \varphi \cdot \sin \theta \cdot \sin \psi-\sin \varphi \cdot \cos \psi) \cdot \frac{u_{1}}{m} \\
& \ddot{z}=g-(\cos \varphi \cdot \cos \theta) \cdot \frac{u_{1}}{m} \\
& \ddot{\varphi}=\dot{\theta} \dot{\psi}\left(\frac{I_{y}-I_{z}}{I_{x}}\right)-\frac{I_{R}}{I_{x}} \dot{\theta} g(u)+\frac{L}{I_{x}} u_{2} \\
& \ddot{\theta}=\dot{\phi} \dot{\psi}\left(\frac{I_{z}-I_{x}}{I_{y}}\right)+\frac{I_{R}}{I_{y}} \dot{\varphi} g(u)+\frac{L}{I_{y}} u_{3} \\
& \ddot{\psi}=\dot{\theta} \dot{\varphi}\left(\frac{I_{x}-I_{y}}{I_{z}}\right)+\frac{1}{I_{z}} u_{4}
\end{aligned}
$$

Equations (11) to (16) represent the full mathematical model of the quadrotor.

\section{ATTITUDE CONTROL ALGORITHM DESIGN}

In this section two controllers for the stabilization of the quadrotor will be presented; the proportional Integral Derivative (PID) controller, and a fuzzy system that tunes the PID gains.

As mentioned before the inputs chose like equations (6) to (9). Where $u_{1}$ controls the motion along the z-axis, $u_{2}$ controls rotation along the x-axis (roll angle), ${ }^{u_{a}}$ controls the rotation along the y-axis (pitch angle) and $u_{4}$ controls rotation along the $\mathrm{z}$-axis (yaw angle). The designed controllers should set values to $u_{i}$ parameters which determines the four rotor speed parameters $\omega_{i}$ by (6) to (9).

\subsection{Classical PID Controller}

Due to their simple structure and robust performance, proportional-integral-derivative (PID) controllers are the most commonly used controllers in industrial process control. The equation of a PID controller has the following form:

$$
u(t)=K_{p} e(t)+K_{I} \int_{0}^{t} e(t) d \tau+K_{D} \frac{d}{d t} e(t)
$$

Where $K_{P}, K_{I}$ and $K_{D}$ are called the propositional, integral and derivative gains, respectively. For adjusting the controller parameters the Ziegler-Nichols method will be used and response of the system with this adjusting will be showed in the result section. 


\subsection{Fuzzy System For Tuning The PID Gains}

The success of the PID controller depends on an appropriate choice of the PID gains. Tuning the PID gains to optimize performance is not a trivial task. In practice, the PID gains are usually tuned by experienced human experts based on some "rule of thumb". In the next we will first determine a set of tuning rules (fuzzy IF-THEN rules) for the PID gains, and then combine these rules into a fuzzy system that is used to adjust the PID gains on-line [15].

A fuzzy PID controller takes the classical PID controller as the foundation which uses the fuzzy reasoning and variable universe of discourse to regulate the PID gains. The characteristics of a fuzzy system such as robustness and adaptability can be successfully incorporated into the controlling method for better tuning of PID gains.

The term self-tuning refers to the characteristics of the controller to tune its controlling parameters on-line automatically so as to have the most suitable values of those gains which result in optimization of the process output. Fuzzy self-tuning PID controller works on the control rules design on the basis of theoretical and experience analysis. Therefore, it can tune the gains $K_{P}, K_{I}$ and $K_{D}$ by adjusting the other controlling parameters and factors on-line. This, in result makes the precision of overall control higher and hence gives a better performance than the classical PID controller or a simple fuzzy PID controller without self-tuning ability.

The self-tuning fuzzy PID controller, which takes error "e" and rate of change-in-error "ed" as the input to the controller makes use of the fuzzy controller rules to modify PID gains on-line. The self-tuning the PID controller refers to finding the fuzzy relationship between the three gains of PID, $K_{P}, K_{I}$ and $K_{D}$ and "e" and "ed", and according to the principle of fuzzy control modifying the three gains in order to meet different requirements for control gains when "e" and "ed" are different and making the control object produce a good dynamic and static performance. For selecting the language variables of "e" and "ed", is choose seven fuzzy values (NB, NM, NS, ZO, PS, PM, PB) which NB denotes Negative Big, NM denotes Negative Medium, Negative Small (NS), Zero (ZO), Positive Small (PS), Positive Medium (PM) and PB denotes Positive Big, and for the outputs we are choose seven fuzzy values (VVS, VS, S, M, B, VB, VVB) which VVS denotes Very Very Small, Very Small (VS), Small (S), Medium (M), Big (B), Very Big (VB) and VVB denotes Very Very Big.

The figure. 2 shows the block diagram of a fuzzy self-tuning PID controller. As it can be seen from the block diagram, the fuzzy system takes two inputs (e and ed) and gives three outputs ( $K_{p}$, $K_{I}, K_{D}$ ) (Figure. 3). This block diagram just shows control of one degree of freedom of a quadrotor and we are used two other controllers like this controller for attitude control of quadrotor.

The membership functions of the all inputs and outputs have been chosen identical. Figure. 4 shows these membership functions. This membership functions are combined of triangular and Gaussian. The width of the fuzzy sets used for controllers are not same and they have been determined by trial and error experience. The width of the fuzzy sets for output, $K_{p}$, have been chosen [0.2 0.7], $K_{I}$, have been chosen [0.001 0.01] and for $K_{D}$, [0.1 0.15$]$. And for inputs, the range for the error have been chosen [-1 1$]$ and for error rate have been chosen $\quad\left[\begin{array}{ll}-10 & 10\end{array}\right]$ and if these inputs put out of these ranges we are used a saturation for put those in the range.

The set of linguistic rules is the essential part of a fuzzy controller. In many cases it is easy to translate an expert's experience into these rules and any number of such rules can be created to 
International Journal of Control Theory and Computer Modeling (IJCTCM) Vol.7, No.1/2, April 2017

define the actions of the controller. In some other cases this rules can come from some trial and error approaches. Table. 1 and Table. 2 shows the fuzzy rules for tuning the PID gains.

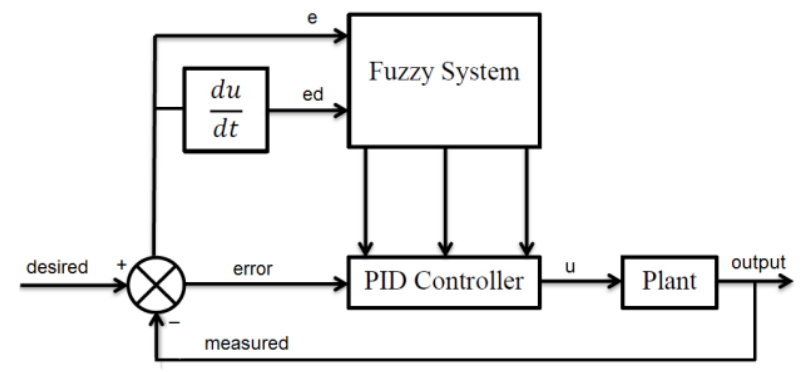

Figure. 2 Basic structure of plant whit controller

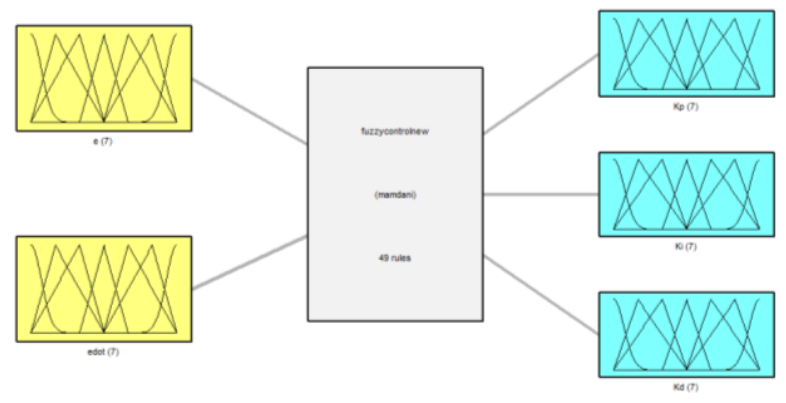

Figure. 3 Basic structure of a fuzzy controller

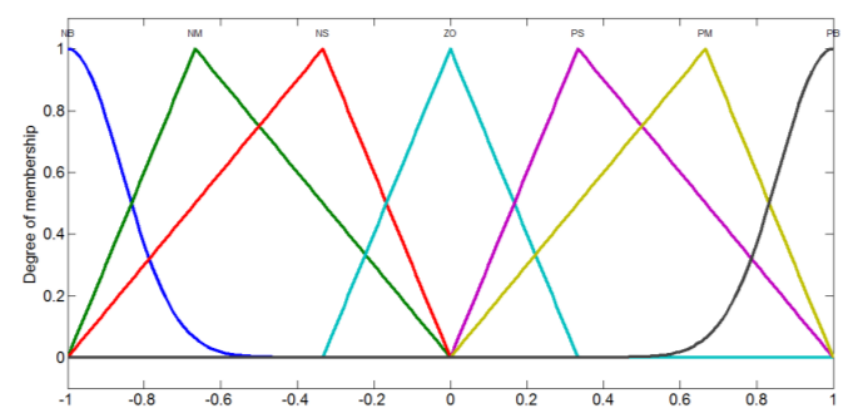

Figure.4 Membership functions for the all inputs and outputs

Table 1. Fuzzy rules for ${ }^{K_{z}}$ and ${ }^{K_{I}}$

\begin{tabular}{|c|c|c|c|c|c|c|c|c|}
\hline & \multicolumn{8}{|c|}{ ERROR } \\
\hline \multirow{8}{*}{ 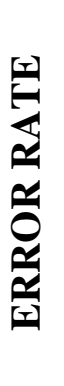 } & & NB & NM & NS & $\mathbf{Z O}$ & PS & PM & PB \\
\hline & NB & $\mathrm{M}$ & $S$ & VS & VVS & VS & $S$ & $\mathrm{M}$ \\
\hline & $\mathbf{N M}$ & B & $\mathrm{M}$ & $\mathrm{S}$ & $\mathrm{VS}$ & $S$ & $\mathrm{M}$ & B \\
\hline & NS & $\mathrm{VB}$ & $\mathrm{B}$ & $\mathrm{M}$ & $S$ & $\mathrm{M}$ & $\mathrm{B}$ & VB \\
\hline & Zo & VVB & VB & B & $\mathrm{M}$ & B & VB & VVB \\
\hline & PS & VB & $\mathrm{B}$ & $\mathrm{M}$ & $S$ & $\mathrm{M}$ & $\mathrm{B}$ & VB \\
\hline & $\mathbf{P M}$ & $\mathrm{B}$ & $\mathrm{M}$ & $\mathrm{S}$ & VS & $\mathrm{S}$ & $\mathrm{M}$ & $\mathrm{B}$ \\
\hline & PB & $\mathrm{M}$ & $S$ & VS & VVS & VS & $S$ & $\mathrm{M}$ \\
\hline
\end{tabular}


Table 2. Fuzzy rules for $K_{\infty}$

\begin{tabular}{|c|c|c|c|c|c|c|c|c|}
\hline & \multicolumn{8}{|c|}{ ERROR } \\
\hline \multirow{8}{*}{ 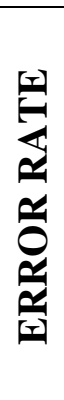 } & & NB & $\mathbf{N M}$ & NS & $\mathbf{Z O}$ & PS & $\mathbf{P M}$ & PB \\
\hline & NB & $\mathrm{M}$ & B & VB & VVB & VB & $B$ & $\mathrm{M}$ \\
\hline & $\mathbf{N M}$ & $S$ & $\mathrm{M}$ & B & VB & B & $\mathrm{M}$ & $S$ \\
\hline & NS & VS & $S$ & $\mathrm{M}$ & B & $\mathrm{M}$ & $S$ & VS \\
\hline & Zo & VVS & VS & $S$ & $\mathrm{M}$ & $S$ & VS & VVS \\
\hline & PS & VS & $S$ & $\mathrm{M}$ & B & $\mathrm{M}$ & $\mathrm{S}$ & VS \\
\hline & $\mathbf{P M}$ & $S$ & $\mathrm{M}$ & B & VB & $\mathrm{B}$ & $\mathrm{M}$ & $S$ \\
\hline & PB & $\mathrm{M}$ & B & VB & VVB & VB & B & $\mathrm{M}$ \\
\hline
\end{tabular}

The rules presented at Tables. 1 and 2 can be read as follows:

For example, IF the error is NB and the error rate is PS THEN $K_{P}$ is VB and $K_{I}$ is VB and $K_{D}$ is VS. The output of the fuzzy system logic is fuzzy.

We cannot provide these fuzzy outputs to a dynamical system as control inputs directly. Defuzzification process is needed to convert these fuzzy outputs to numbers that can represent the fuzzy output. The control signal should be continuous, any variation in input should not produce big changes in output signal. The defuzzification algorithm should be clear and the process to determine the output signal should be identified clearly. Also, the defuzzification should be logical, should have high membership degree and it should correspond to the approximately middle of the graph. For the reason discussed above we have selected center average defuzzifier for the controllers.

\section{ReSUlts And Simulation Study}

The mathematical dynamical model of the quadrotor vehicle as well as the controllers have been developed in Matlab Simulink for simulation. The goal of this analysis is to test how well the controllers can stabilize the quadrotor. We are supposed the quadrotor starts with 6 (rad) pitch, 3 (rad) roll and -2 (rad) yaw angle in the hovering mode. The quadrotor is commanded to change these angles to zero and stable itself. Both of the controllers are run on these two scenarios and the results are compared.

For adjusting classical PID controller gains we are used Ziegler-Nichols method. Due to this method we are choose for the PID gains.

$$
\begin{aligned}
& K_{p}=0.3, K_{I}=0.475 \text { and } K_{D}= \\
& 0.11875
\end{aligned}
$$

And by using the fuzzy system for tuning the PID gains, the PID gains change along the simulation that you can see results of this changing in Figure. 5, for example changing the PID gains in roll angle.

The results of two methods for three Euler angles are showed in Figure. 6 to Figure. 8. In Figure. 6 you can see response of the quadrotor for the roll angle, Figure. 7 shows the response for the pitch angle and Figure. 8 shows response of the quadrotor for the yaw angle. Additionally figures show differences between two methods, classical PID controller and fuzzy PID controller. 
From the figures clearly can be understand that by using the fuzzy system for tuning the PID gains the performances improved. Overshoot in three Euler angles is less than classical PID gains. The setting time of three angles by using the fuzzy system, obviously decreased. In the yaw angle there is no big different between two methods but in it the overshoot decreased. In two other angles you can see big different between classical PID controller and fuzzy PID controller.

\section{EXPERIMENTAL RESULTS}

After the design of controller, this controller implemented on a real quadrotor. The main rig of this test setup is a quadrotor equipped with IMU sensor type GY80 measures the roll, pitch and yaw angle of quadrotor $(\varphi, \theta, \psi)$. Moreover, an Arduino Board (UNO) has been used as a microcontroller which its duties are sending, receiving and operating data in order to stabilize the quadrotor (Figure.9).

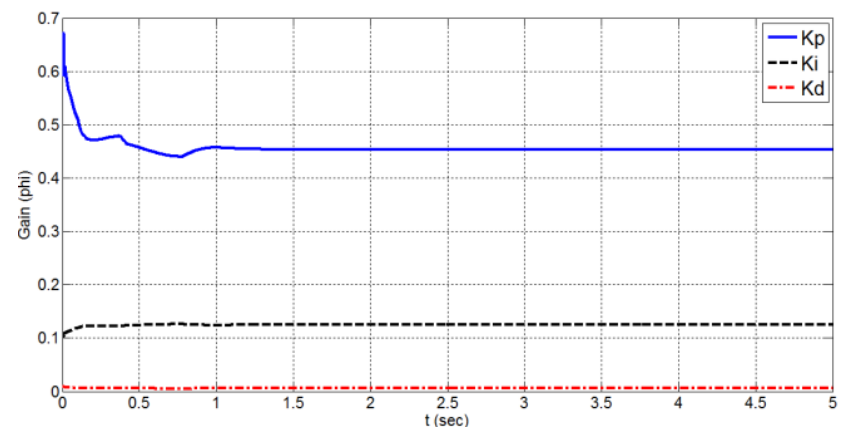

Figure. 5 Changing the PID gains along the simulation for roll angle

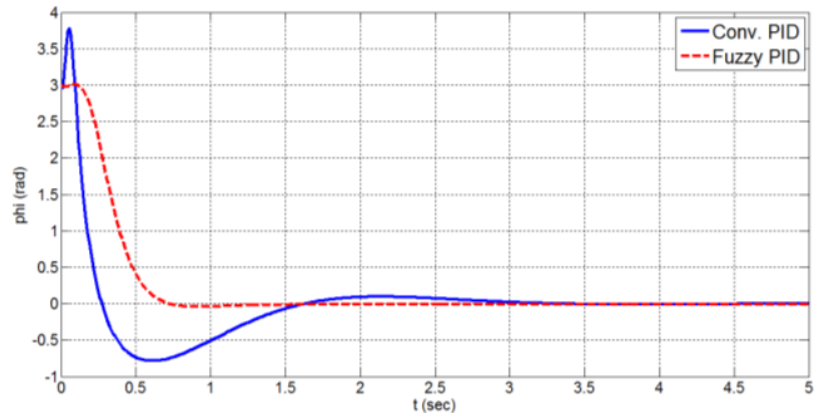

Figure. 6 The roll angle control of the quadrotor using classical PID (solid line) and fuzzy PID (dashed line) controllers

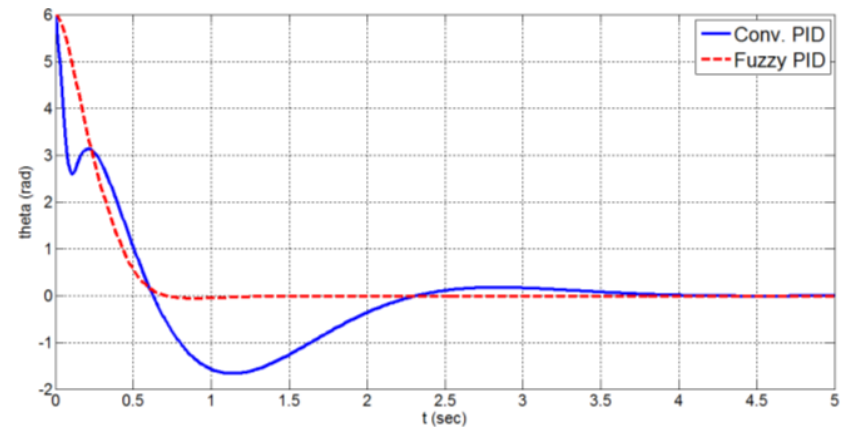

Figure. 7 The pitch angle control of the quadrotor using classical PID (solid line) and fuzzy PID (dashed line) controllers 


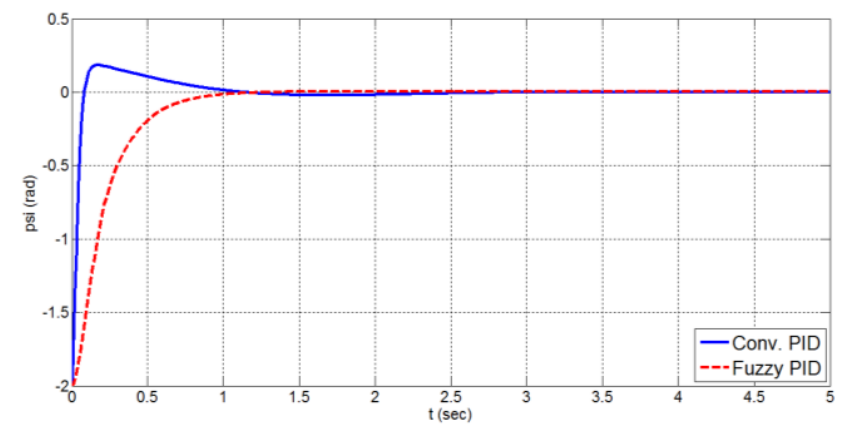

Figure. 8 The yaw angle control of the quadrotor using classical PID (solid line) and fuzzy PID (dashed line) controllers

In order to eliminate noise from IMU data, $260 \mathrm{~Hz}$ and $256 \mathrm{~Hz}$ low pass filter are applied to the accelerometer and gyro data correspondingly. Generally, the accelerometer is very noisy while the gyro drifts over time. In other words, the gyroscope data can be used on a short term while the accelerometer data can be utilized on a long term. To treat this, a Kalman filter is then designed and implemented in which a combination of accelerometer and gyro data are employed to achieve a better roll, pitch and yaw angle estimation along with removing unwanted residual noise. Figure. 10 and Figure. 11 shows performance of applying filter to have satisfactory data of the roll angle.

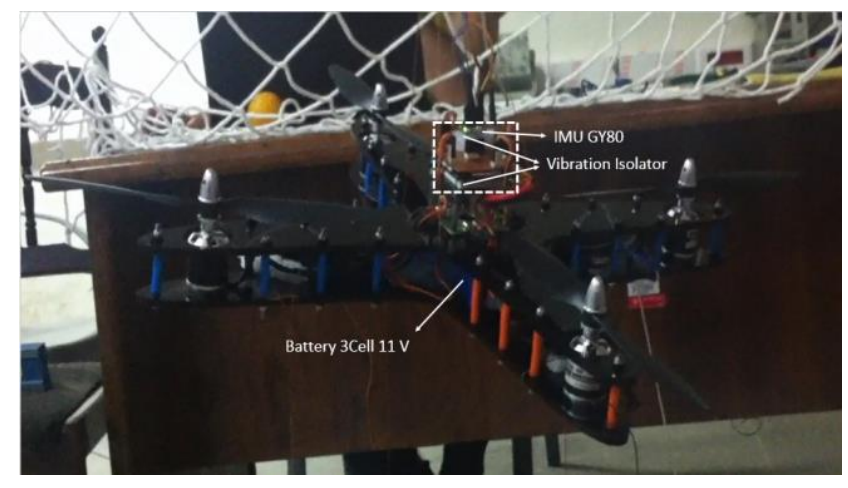

Figure. 9 Prototype equipped quadrotor

The measured roll, pitch and yaw angles by IMU sensor, are transferred to Arduino board. After some numerical computations like filtering and buffering of sensor data, the values of 3 Euler angles are sent to computer. This information is employed in MATLAB software and using them, suitable control signal is computed by designed algorithm. The algorithm output (PID controller parameters) comes back to Arduino board and then using the electrical speed controller, the quadrotor's attitude is changed (Figure. 12).

Two experimental tests are performed to evaluate the effectiveness of the fabricated Fuzzy-PID controller. 
International Journal of Control Theory and Computer Modeling (IJCTCM) Vol.7, No.1/2, April 2017

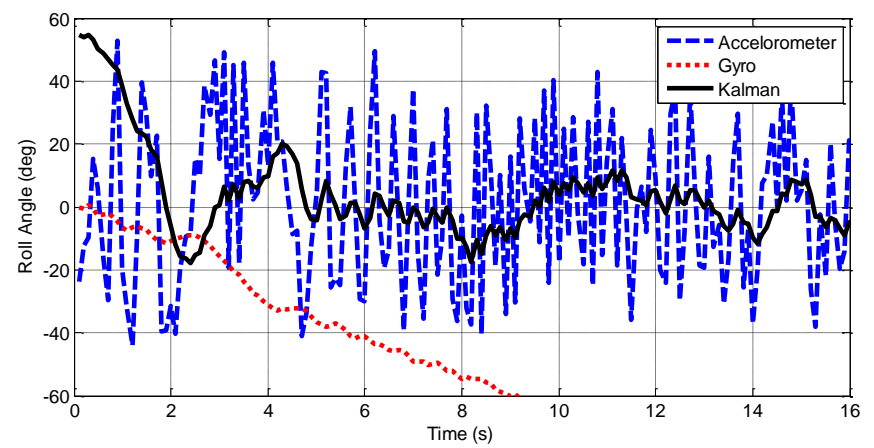

Figure. 10 Accelerometer, Gyro Roll data before applying low pass filter and roll angle with kalman filter

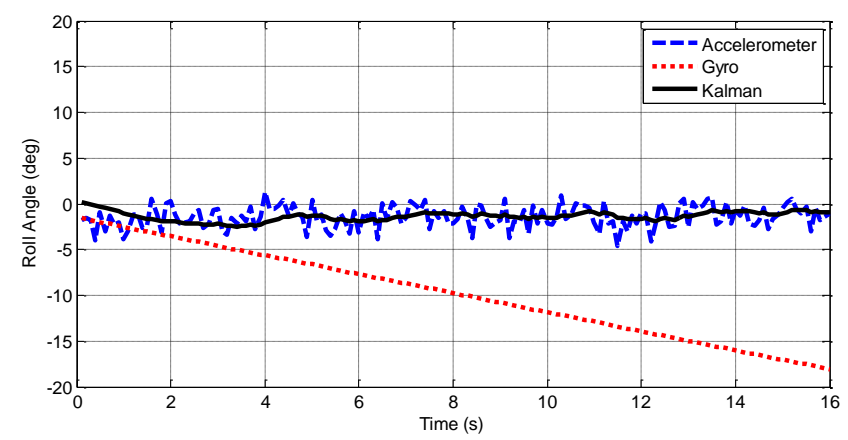

Figure. 11 Accelerometer, Gyro Roll data after applying low pass filter and roll angle with kalman filter

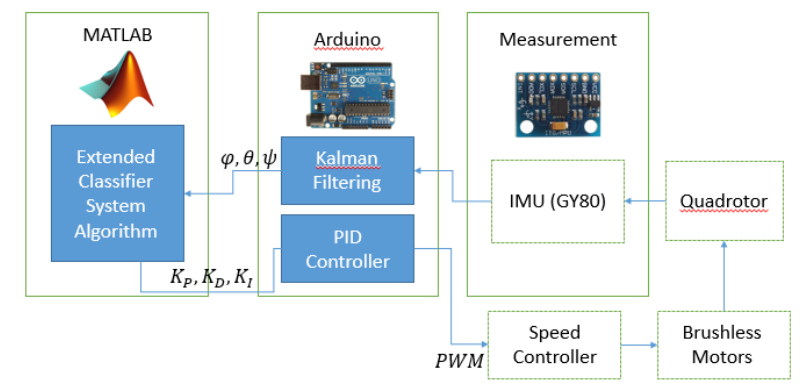

Figure. 12 Exchanging data between different equipments.

\subsection{FIRST TEST}

We get the quadrotor in a situation such that pitch angle starts at $+20(\mathrm{deg})$ in hovering mode. The quadrotor is commanded to change these angles to zero and stable itself. In the below figures you will see results of this test for controlling roll and pitch angle. In first two figures shows changes of PID parameters during the test for both roll and pitch angles (Figure. 13 and 14). Other two figures shows the result of controller performance (Figure. 15 and 16).

It is necessary to say for stabilize of IMU data we should spend about 2 seconds. Thus in these figures quadrotor starts after 2.2 seconds.

\subsection{SECOND TEST}

In the final test, has been tried to show performance of the presented controller, when two heavy weights have been connected to corners of the quadrotor. These two weights make the quadrotor 
be unstable. A 370 (gr) weight has been connected to one corner of the quadrotor to distort the roll angle and a 400 (gr) weight will be distorted the pitch angle. Now the controller will be tried to stable the quadrotor. Results of this test showed in figure. 17.

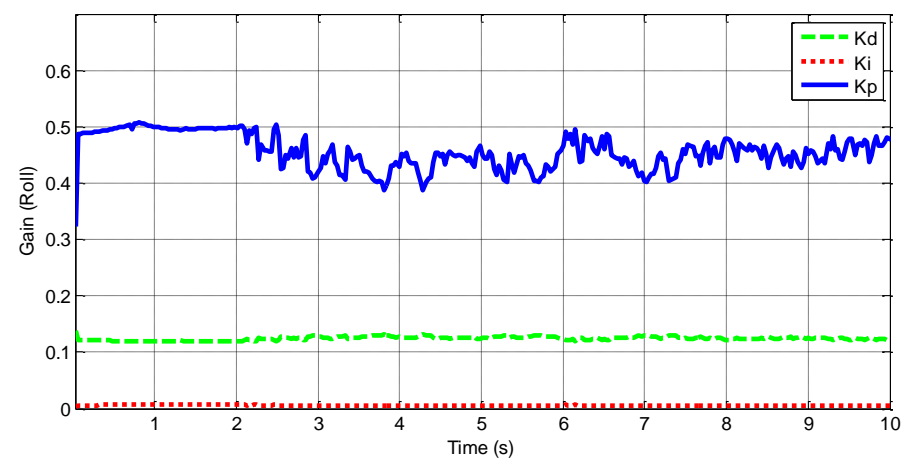

Figure. 13 Changing the PID gains along the test for roll angle

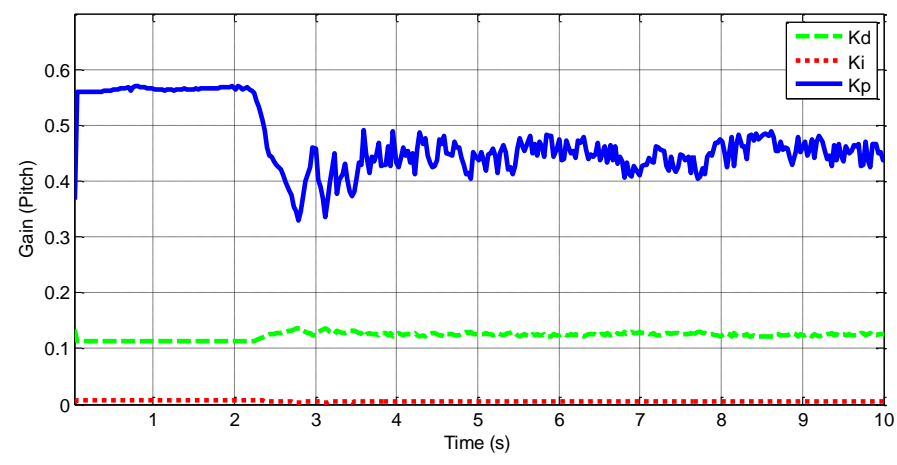

Figure. 14 Changing the PID gains along the test for pitch angle

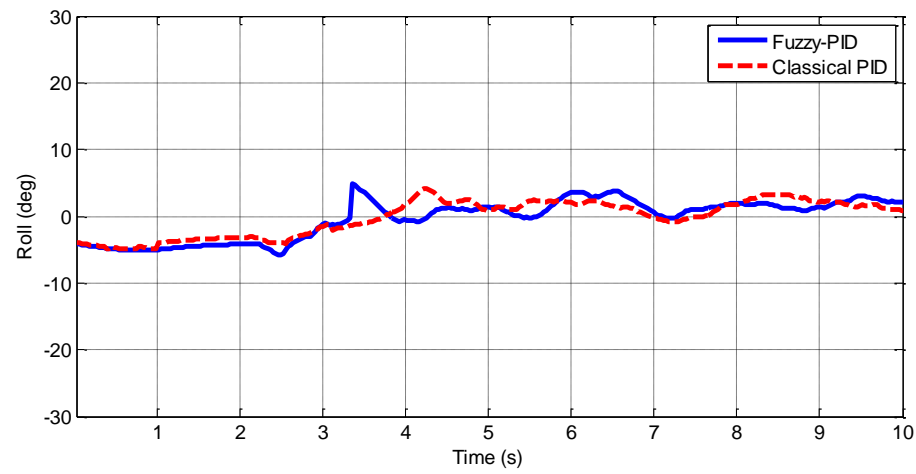

Figure. 15 The roll angle control of the quadrotor by using fuzzy PID _ angle (solid line) and classical PID controller (dashed line) 


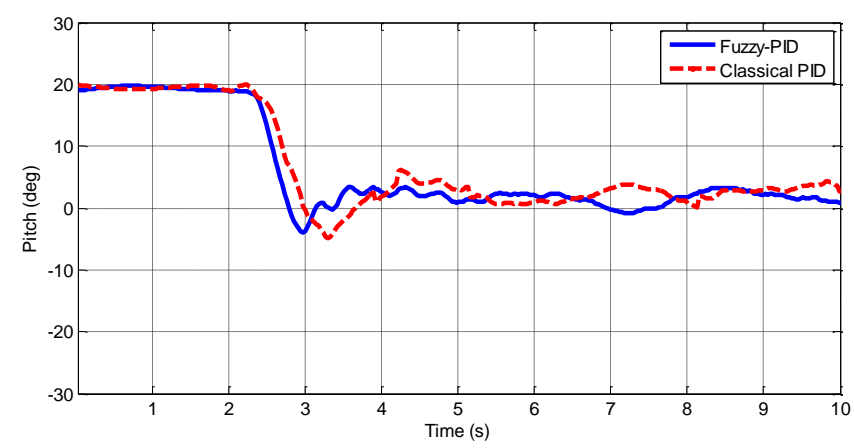

Figure. 16 The pitch angle control of the quadrotor by using fuzzy PID _ angle (solid line) and classical PID controller (dashed line)

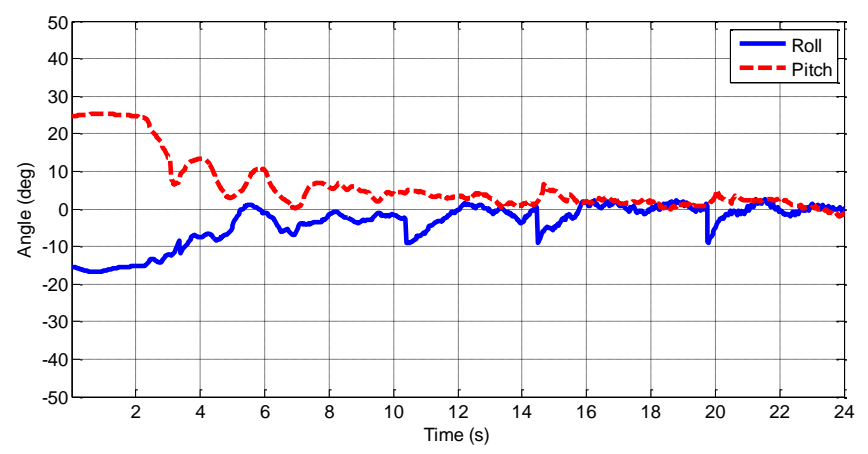

Figure. 17 The roll (solid line) and pitch (dashed line) angle control of the quadrotor by using fuzzy PID

\section{CONCLUSION}

In this paper, modelling, a classical PID controller and a fuzzy supervisory controller for tuning the PID controller gains has been presented. Although fuzzy control of dynamical system has been presented extensively in literature, application of this technology to quadrotor control is quite new. In the first step we modeled the quadrotor with Euler-Newton equations. In the next we used a classical PID controller to attitude control of the quadrotor. For first time we used Ziegler-Nichols method for adjusting the PID gains and after that introduced a fuzzy supervisory controller for tuning the PID gains and attached this controller to the classical PID controller. We used three sets of these controllers for control of the quadrotor's three Euler angles. Various simulations based on the Matlab Simulink, were performed to test and compare the two control algorithms. When comparing these two types of controller we should note that the selection of the membership functions and the rules of the fuzzy controller are based on the experience of the designer and furthermore using a few trial and error approach.

The dependence of the fuzzy controller on the quadrotor model is much less than the classical controllers used. The fuzzy controller is nonlinear and it is thus more suitable for nonlinear system control. The control performances of the fuzzy PID controller was greatly better than the classical PID controller as you can see in the result section. The biggest advantages of the fuzzy PID controller is eliminating overshoots than the big overshoots in the classical PID controller and the other one is smaller setting time than the classical PID controller.

In the next step we implemented this control methods to the real quadrotor and performed some tests to show abilities of the presented controller. Results of this tests showed and you can see that this results verify the simulation results. 
In next studies we will develop some other control methods to the quadrotor both in simulation and experiment and will compare this control methods and will select the best controller in the real experiment tests.

\section{APPENDIX}

Some constant's values which used in the simulation of the quadrotor are showed in Table. 3. This constant's values selected from a real quadrotor in laboratory of mechatronic in the University of Tehran.

Table 3 Table of constants

\begin{tabular}{|c|c|c|}
\hline Symbol & Description and unit & value \\
\hline$\varphi$ & Roll angle (rad) & _ \\
\hline$\theta$ & Pitch angle (rad) & _- \\
\hline$\psi$ & Yaw angle (rad) & \\
\hline $\mathrm{m}$ & Mass of the quadrotor $(\mathrm{Kg})$ & 1.25 \\
\hline l & $\begin{array}{l}\text { Center of quadrotor to center of propeller } \\
\text { distance }(\mathrm{m})\end{array}$ & 0.2 \\
\hline$I_{x}$ & $\begin{array}{l}\text { Body moment of inertia around the } \mathrm{x} \text {-axis } \\
\left(\mathrm{Nms}^{\mathbf{2}}\right)\end{array}$ & 0.002353 \\
\hline L & $\begin{array}{l}\text { Body moment of inertia around the y-axis } \\
\left(\text { Nms }^{2}\right)\end{array}$ & 0.002353 \\
\hline$I_{x}$ & $\begin{array}{l}\text { Body moment of inertia around the z-axis } \\
\left(\text { Nms }^{z}\right)\end{array}$ & 0.004706 \\
\hline$b$ & Thrust factor & $2.92 \times 10^{-2}$ \\
\hline$d$ & Drag factor & $1.12 \times 10^{-7}$ \\
\hline$I_{R}$ & Rotor inertia $\left(N m s^{2}\right)$ & $2 \times 10^{-5}$ \\
\hline
\end{tabular}

\section{REFERENCES}

[1] Gessow and G. Myers, Aerodynamics of the helicopter, Fredrick Ungar Publishing Co, New York, (1967).

[2] J. G. Leishman, Principles of Helicopter Aerodynamics, Cambridge University Press, (2000).

[3] M. J. Hirschberg, The American Helicopter: An overview of Helicopter Developments in America 1908-1999, (2000).

[4] P. Castillo, R. Lozano, and A. E. Dzul, Modeling and Control of Mini-flying Machines, Advances in Industrial Control Series, ISSN 1430-9491, Springer, (2005).

[5] H. Y. Chao, Y. C. Cao, and Y. Q. Chen, "Autopilots for small unmanned aerial vehicles: a survey," International Journal of Control, Automation, and Systems, vol. 8, no. 1, pp. 36-44, (2010).

[6] D. Lee, I. Kaminer, V. Dobrokhodov, and K. Jones, "Autonomous feature following for visual surveillance using a small unmanned aerial vehicle with gimbaled camera system," International Journal of Control, Automation, and Systems, vol. 8, no. 5, pp. 957-966, (2010).

[7] D. Han, J. Kim, C. Min, S. Jo, J. Kim, and D. Lee, "Development of unmanned aerial vehicle (UAV) system with waypoint tracking and vision-based reconnaissance," International Journal of Control, Automation, and Systems, vol. 8, no. 5, pp. 1091-1099, (2010).

[8] E. Altuğ, J. P. Ostrowski, and C. J. Taylor, "Control of a quadrotor helicopter using dual camera visual feedback," International Journal of Robotics Research, vol. 24, no. 5,pp. 329-341, (2005).

[9] D. Suter, T. Hamel, and R. Mahony, "Visual servo control using homography estimation for the stabilization of an X4-flyer," Proc. Of the 41th IEEE conf. on Decision and control, PP. 2872-2877, (2002).

[10] J. Dunfied, M.Tarbouchi, and G. Labonte, "Neural network based control of a four rotor helicopter," Proc. of IEEE Int. Conf. on Industrial Technology, PP. 1543-1548, (2004).

[11] M. G. Earl and R. D'Andra, "Real-time attitude estimation techniques applied to a four rotor helicopter," Proc. of IEEE conf. on Decision and Control, PP. 3956-3961, (2004). 
[12] D. Lee, H. J. Kim, and S. Sastry, "Feedback linearization vs. adaptive sliding mode control for a quadrotor helicopter," International Journal of Control, Automation, and Systems, vol. 7, no. 3, pp. 419-428, (2009).

[13] B. Erginer, and E. Altuğ, "Design and implementation of a hybrid fuzzy logic controller for a quadrotor VTOL vehicle," International Journal of Control, Automation, and Systems, vol. 10, no. 1, pp. 61-70, (2012).

[14] A. Sharma, and A. Barve, "Controlling of quadrotor UAV using PID controller and fuzzy logic controller," International Journal of Electrical, Electronics and computer Engineering, vol. 1, no. 2, pp. 38-41, (2012).

[15] L.-X. Wang, A Course in Fuzzy System and Control, Prentice Hall, 1997.

[16] C.-C. Lee, "Fuzzy logic in control systems: fuzzy logic controller-part I, ” IEEE Trans. On System, Man, and Cybernetics, vol. 20, no. 2, pp. 404-418, (1990).

[17] L. Reznik, Fuzzy Controllers Handbook, (1997).

[18] E. H. Fung, Y. Wong, Y. Ma, C. M. Yuen, and W. Wong, "Smart hanger dynamic modeling and fuzzy control design," International Journal of Control, Automation, and Systems, vol. 9, no. 4, pp. 691-700, (2011).

[19] A. Hafaifa, F. Laaouad, and K.Laroussi, "A numerical structural approach to surge detection and isolation in compression systems using fuzzy logic controller," International Journal of Control, Automation, and Systems, vol. 9, no. 1, pp. 69-79, (2011).

[20] A. Catena, C. D. Melita, G. Muscato,"Automatic Tuning Architecture for the Navigation Control Loops of Unmanned Aerial Vhicles," Journal of Intelligent and Robotic Systems, vol. 73, no. 1, pp. 413-427, (2014).

[21] M. Sugeno, Development of an Intelligent Unmanned helicopter, at the Fuzzy Modeling and Control, CRC Press, Boca Raton, (1999).

[22] B. Kadmiry, and D. Driankov, "Fuzzy control of an autonomous helicopter," IFSA World Congress, vol. 5, Canada, pp. 2797-2802, (2001).

[23] C. Cavalcante, J. Cardoso, J. G. Ramos, and O. R. Nerves, "Design and tuning of a helicopter fuzzy controller," Proc. of IEEE Int. Conference on Fuzzy Systems, vol. 3, pp. 1549-1554, (1995).

[24] N. I. Vitzilaios, and N. C. Tsourveloudis, "An experimental test bed for small unmanned helicopters," Journal of Intelligent and Robotic Systems, vol. 54, pp. 769-794, May (2009).

[25] R. D. Garcia, and K. P. Valavanis, "The implementation of an autonomous helicopter test bed," Journal of Intelligent and Robotic Systems, vol. 54, Issue 1-3, pp. 423-454, March (2009).

[26] H. Voos, "Nonlinear Control of a Quadrotor Micro-UAV Using Feedback-Linearization," IEEE International Conference on Mechatronics, pp. 1-6, (2009).

[27] E. Abbasi, M. Mahjoob, " Controlling of Quadrotor UAV using a Fuzzy System for Tuning the PID Gains in Hovering Mode.", Oral presentation at Advances in Control Engineering Conference (ACE 2013), Istanbul, Turkey, (2013).

[28] E. Abbasi, " Quadrotor UAV guidance of ground moving target tracking.", Journal of Advances in Computer Engineering and Technology, Volume2, Issue 2, Page 37-44, (2016).

[29] Semwal, Vijay Bhaskar, and Gora Chand Nandi. "Generation of Joint Trajectories Using Hybrid Automate-Based Model: A Rocking Block-Based Approach." IEEE Sensors Journal 16.14 (2016): 5805-5816.

[30] Nandi, Gora Chand, et al. "Modeling bipedal locomotion trajectories using hybrid automata." Region 10 Conference (TENCON), 2016 IEEE. IEEE, 2016.

[31] Semwal, Vijay Bhaskar, et al. "Design of Vector Field for Different Subphases of Gait and Regeneration of Gait Pattern." IEEE Transactions on Automation Science and Engineering (2016).

[32] Semwal, Vijay Bhaskar, et al. "An optimized feature selection technique based on incremental feature analysis for bio-metric gait data classification."Multimedia Tools and Applications: 1-19.

[33] Semwal, Vijay Bhaskar, Pavan Chakraborty, and Gora Chand Nandi. "Less computationally intensive fuzzy logic (type-1)-based controller for humanoid push recovery." Robotics and Autonomous Systems 63 (2015): 122-135.

\section{Authors}

Ehsan Abbasi received M.s degree in Mechanical Engineering from University of Tehran, Tehran, Iran in 2014. Now he studies at Isfahan University of Technology for receiving PhD degree. His current research interests include controlling of dynamical systems and robotics and mechatronics.

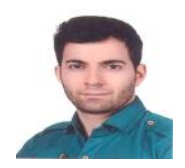

